\title{
Optimized Energy-efficient Load Balance Routing Protocol for Wireless Mesh Networks
}

\author{
M Kiran Sastry, Arshad Ahmad Khan Mohammad, Arif Mohammad Abdul \\ Assistant Professor, Department of Computer Science and Engineering \\ GITAM (Deemed to be University) \\ Hyderabad, India
}

\begin{abstract}
Wireless mesh network (WMN) technology gains the user attractions due to its deployment flexibility. The main challenging task in the WMN is the provision of Quality of Service due to its unbalanced traffic in communication. Moreover, the recent advancement in wireless technology has fueled the user's attraction towards the delay-sensitive services, which in turn additional impact on WMN to provide QoS. The paper aims to provide the QoS in the WMN by load balancing, and energy efficiency. In literature, various mechanisms have been designed to address the issue, but they fail to achieve the optimal solution in terms of throughput and energy efficiency. Thus, the work aims to design the energy-efficient load balancing routing metric to address the limitations of existing conventional methods. Load balancing is accomplished by the selection of noncongested nodes, and energy efficiency is attained by the selection of the greatest packet processing capable nodes for communication. Both non-congested and greatest packet processing capable nodes are used to compute the route between source and destination. The performance of the proposed routing protocol is analyzed by network simulator and the results outperformed in comparison with recent existing methods.
\end{abstract}

Keywords-Wireless mesh network; routing; energy efficiency; optimization

\section{INTRODUCTION}

Wireless mesh networks (WMNs) technology [1] is the advancement of mobile ad hoc networks [3] that aims to provide greater bandwidth and less cost communication services to the users. WMNs are composed of mesh clients, routers, and gateways, where routers interconnect one another through a mesh topology [1] and communicate in a multi-hop manner [3]. The mesh clients in WMNs communicate in a peer-to-peer manner [3]. The mesh clients are user apparatus, and they are connected to the internet via mesh routers and gateway, where the gateways are special types of routers. The Fig. 1 shows the WMNs architecture.

The characteristics of WMNs are cost effective, fast deployment, and greater bandwidth, these characteristics have been attracting the users to select this technology for communication. However, one of the major challenges in WMNs is to provide QoS communication. The reason behind this limitation is unbalanced traffic and further this technology is not designed to work effectively in a congested environment. Thus, some of the nodes in the network take heavy traffic than the other nodes and cause the congestion, and it is known as unbalanced traffic distribution. It causes the packets drop in the network at routing layer, which interns negatively impacts the system performance in terms of energy efficiency, delivery of packets, and QoS. The paper aims to design the routing protocol to avoid the packets drop at network layer.

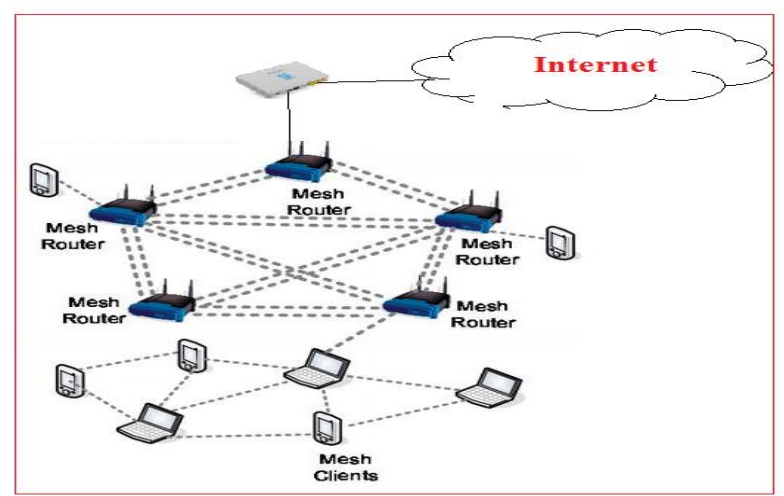

Fig. 1. Wireless Mesh Networks Architecture.

\section{LITERATURE REVIEW}

In literature, various routing mechanisms have been designed to adder the issue of unbalanced traffic distribution in WMN by traffic re-routing [1]. However, they do not consider the load status at the node buffer. Thus, the queue at the some of the mesh nodes buffer increases and causes the congestion and further negatively impact on the overall network performance.

A selective load balancing (ViLBAS) [5] technique is designed to address the congestion at mesh nodes during video delivery. This technique enhances network performance in terms of packet delivery. However, it is not designed for all delay-sensitive traffic conditions. Similarly, an efficient load balancing routing protocol designed by work [6], to achieve the QoS in terms of network lifetime, minimum delay, and guaranteed bandwidth. Work [7] finds that the VILBAS [5] and work [6] are not attained effective load balancing in WSN. The work [8] designed the Load balanced coding aware multipath routing to balance the traffic in the network. The performance results show that the work [8] achieves the load balancing efficiency with increased routing overhead, delay, and energy consumption. The work [9] designed the interface shifting or load balancing method to resolve the problem of energy consumption along with load balancing.

A review of various mechanisms developed for load balancing and energy efficiency was analyzed in work [6] and concluded that there is a requirement of the routing protocol 
which needs to attain the energy efficiency with load balancing to provide the QoS in WMNs. Thus, the aim is to provide the QoS in the WMN by load balancing with energy-efficiency. The major contributions of the work are as follows:

1) Load balancing in $\mathrm{WMN}$ is achieved by considering the queue constructed at node buffer due to the network layer.

2) Energy efficiency is achieved by considering the packet processing ability of node concerning residual energy.

3) The selection of the route between communication entities by maximizing the Energy efficiency and avoiding the congestion.

The reminder of the paper is organized as follows.

Next section describes the Load Balancing in WMNs by computing queuing at network layer, further section explains with Energy efficiency by packet processing ability of the nodes. The proposed energy-efficient load balance routing for multi-radio WNNs is explained in Section 5. The paper ends with conclusion followed by future work.

\section{LOAD BALANCING IN WMNS BY COMPUTING QUEUING AT NETWORK LAYER}

Load balancing in communication network is the procedure to distribute the traffic load across the multiple entities [18]. Load balancing approach is used to enhance the network performance and avoid the congestion. In WMNs multi path routing is a common approach, and thus the load balancing is achieved by the process of traffic rerouting form congested entities to uncongested entities. In literature, traffic rerouting is achieved by various routing protocols by different metrics [1]. However, they fail to address the congestion at the node buffer, which negatively impact on the overall network performance.

To extend the performance of load balancing routing algorithms designed for delay-sensitive traffic such as voice, video, and multimedia in WMNs, novel load balancing algorithm designed based on the queuing delay [2]. The algorithm detects the nodes with less queue at its network layer and based on it the algorithm assigns the traffic to the nodes for transmission [10]. The routing metric designed based on the queue at the MAC layer of the node is not sufficient to achieve the QoS for delay-sensitive traffic transmission in WMN. There is a possibility in the network that the packets queued at the network layer of the node buffer, and the delay caused by it is more impact on the network performance in comparison with the delay caused by the MAC layer queue [14]. Thus, to decide the node status regarding its load, the queue at network layer needs to be calculated.

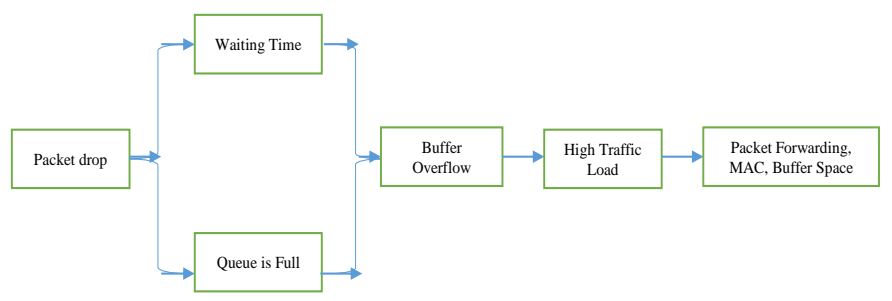

Fig. 2. Packet Drop from Node due to Buffer Overflow Reasons.
Various congestion detection and prevention mechanisms have been designed at the transport layer to overcome the problem of load balancing due to queuing at node buffer [4]. These algorithms are performed well for the wired network, and wireless-infrastructure based networks and cannot directly be applied to the WMNs [3]. Thus, the early congestion prevention algorithm i.e., the queue computation at network layer is needed to overcome the problem of load balancing queuing at node buffer. The load at the node buffer increased due to exceeding high traffic load that is associates with the data forwarding and medium access control [15, 16], and it is shown in Fig. 2. Thus, the traffic load of the nodes needs to be computed at the network layer and according to that the assignment of traffic load. The proposed work computes the queue at the network layer of the node to decide the node status regarding its load. The number of packets is queued at network layer of the node is computed by equation (1).

Averagenewqueue $\left(Q_{\text {avrg }}\right)=$ Instant Queue $*$

(Weighted constant $)+(1-$ Weighted constant $) *$ Avergeoldqueue.

Work considers that every node contains the RED gateway [13] to compute the average queue length of its buffer. It is a low pass filter work on the principle of the exponential weighted moving average. The procedure to calculate the average queue decides the grade of burstiness that would be permitted in the gateway queue. Instead of taking maximum values, we consider the average to prevent the packets drop in advance by setting threshold value, as average queue size above the current threshold. The weighted constant value of equation 1 is computed by low-pass filter time constant value [17] of RED gateway. Threshold value at the network layer queue is computed by equation 2 .

$$
\text { Thresholdbuffer }\left(Q_{T h}\right)=75 \% \text { of buffer size }
$$

If the node Averagenewqueue $\left(Q_{\text {avrg }}\right)$ of the node is above the threshold queue $\left(Q_{T h}\right)$ value, then the node cannot handle further traffic, and known as congested nodes. The proposed algorithm i.e., node selection algorithm shown in Fig. 3 detects congested nodes and avoid them to participate in the communication.

\section{Algorithm 1:- Node selection algorithm}

Procedure to computing the current residual condition of nodes $\left(Q_{\text {avrg }}, Q_{T h}, E P, E_{P t h}\right)$

\{ Compute the $Q_{\text {avrg }}$ from equation 1

if $\left(Q_{\text {avrg }} \leq Q_{T h}\right)$

$\left\{\right.$ Then compute the $E_{P}$ from equation 4

if $\left(E_{P} \geq E_{P t h}\right)$

node participates in routing

else node does not participate in routing $\}$

else Node does not participate in routing \}

Fig. 3. Node Selection Algorithm based on the Congestion and Energy Efficiency. 


\section{Energy EfFiciency by PACKet Processing AbILITy OF THE NODES}

Considering energy for route computation is an active research topic in WMNs, as the nodes in the network are equipped with heterogeneous resources, and they need to act as a router for multi hop communication. To act as router, the node needs to spend its energy to accomplish the routing task. Recent survey shows that that node needs to spend 800-1200 MW of power to receive and transmit the packets [19]. To address the issue, the routing protocols design must consider the energy issue in their routing process. In literature, different routing protocols have been designed to achieve the goal of extension of energy conservation [20]. These protocols broadly categorized as link stability routing, network lifetime extension routing, and energy aware routing. The recent work [21], analyzed these routing protocols, and concluded that they depute the particular path for communication, which leads to heavy traffic towards the nodes, and causes the node to expire due to energy drainage, and drops the packets.

The performance of the WMNs can be enhanced by reducing the packet drop due to the rapid increment of the energy drain of the node. Thus, paper computes the packet processing ability of the node regarding its residual energy and traffic at its node buffer.

Consider all the nodes available in a network consist of energy $(E)$ to process the packets, as nodes in a network act in a peer-to-peer manner. The energy $\left(E_{p i}\right)$ is spent by the node to process the one packet $P_{i}$, which includes packet reception, processing, and transiting energies. Then the residual energy $\left(E_{r}\right)$ of the node is calculated as follows, by equation (2).

$E_{r}=E-E_{p i}$

The residual packet processing ability $\left(E_{P}\right)$ of the node during the time interval $(t)$ is computed as follows:

The residual packet processing ability of the sensor node in its available energy is computed by the following equation 4 .

$E_{P}=\frac{E_{r}}{\left(E_{p i}(t)\right)} \forall \boldsymbol{E}_{\boldsymbol{r}} \geq\left(E_{p i}(\boldsymbol{t})\right)$

The threshold residual packet processing ability of the node is calculated by following equation 5 .

$E_{P t h}=\frac{(75 \%) * E}{\left(E_{p i}(t)\right)}$

If the nodes' residual status of packet processing ability concerning energy is higher than the threshold i.e., $E_{P t h}$ from equation 6 , then the node is considered for communication. The algorithm to consider the node for communication is explained in Fig. 3.

$Q_{t h m}=\left(\frac{h}{2}\right) * Q_{t h}$

Where,

$h=$ hop count from source to destiantion

$Q_{t h m}$

= acceptable threshould for considering the node for routing

\section{ENERGY-EFFICIENT LOAD BALANCE ROUTING FOR MULTI-RADIO WNNS}

Paper considers the WMNs with mobile nodes distributed in the network communication area. The source node wants to communicate with destination through the routing path which must be less congested and energy-efficient to achieve the QoS in communication by enhancing the packet delivery and lifetime of the network. Thus, the paper develops the energyefficient load balance routing protocol for WMNs to select the route with the greater energy-efficient and less congested node for computing the routing path, to enhance the QoS of the network.

The designed routing protocol is an extension of the existing AODV [11] routing protocol. Whenever the source wants to interact with the destination and finds that there is no routing entry is available in the routing-table. Then the source broadcasts the route-request message to compute the route. An intermediate node receives the request message then it checks its average queue by equation 1 and compare it with the threshold queue size equation 2, if it satisfies then it participates in routing otherwise it discards the routing packet. If it satisfies with threshold queue size, then it computes the packet processing ability in terms of energy by equation 4 and compare it with the threshold value of packet processing ability, if it also satisfied then rebroadcasts the route request packet. The same procedure will follow until the route request message reaches the destination. Destination examines all the route request merges received from different intermediate nodes and compute the route as follows.

\section{A. Routing Path selection by Destination node}

The previous sections explain the concept of nodes to participate or non-participate in the routing by average queue size and packet processing ability of the nodes. Now the nodes need to be finalized for routing path from source to destination, the total acceptable queue size for routing path is about $Q_{t h m}$, and it is computed by the following equation.

Each node $n_{i}$ has $Q_{\text {avrg }}$ queue size at its node buffer and having the $E_{P_{i}}$ of residual status of packet processing ability to process the packets. One can avoid the congestion and enhance the energy efficiency in the network, by following considerations:

1) The total queue size of the routing path nodes must not exceed the value of $Q_{t h m}$.

2) The total residual status of packet processing ability of the routing path nodes as much as possible.

To select the nodes for routing path by satisfying above conditions, the work considers the following tuples and considerations.

1) $\left\langle Q_{\text {avrg }_{1}}, Q_{\text {avrg }_{2}}, Q_{\text {avrg }_{3}}, \ldots \ldots Q_{\text {avrg }_{n}}\right\rangle \quad$ and $\left\langle E_{P_{1}}, E_{P_{2}}, E_{P_{3}}, \ldots \ldots . . E_{P_{n}}\right\rangle$.

2) $Q_{t h m}>0$, and work wish to determine the routing path nodes $T \in\left\{n_{1}, n_{2}, n_{3}, \ldots . n_{n}\right\}$ such that; Maximize $\sum_{i \in T} E_{P}$,

And subject to $\sum_{i \in T} Q_{\text {avrg }_{i}} \leq Q_{\text {thm }}$ 
In order to solve the problem of selecting the routing nodes for routing path, work decomposes the problem by constructing the two-dimensional array, as follows.

$V\left[0 \ldots n_{n}, 0 \ldots Q_{t h m}\right] \forall n_{1} \leq n_{i} \leq n_{n}$ and $0 \leq Q_{\text {avrg }_{i}} \leq$ $Q_{t h m}$

The entry of the array $V\left[n_{i}, Q_{\text {avrg }}\right]$ is going to select the nodes $\left\{n_{1}, n_{2}, n_{3}, \ldots . n_{n}\right\}$ by maximizing the packet processing ability and by not exceeding the value of $Q_{t h m}$. The array entry $V\left[n_{n}, Q_{\text {avrg }_{n}}\right]$ provides the routing path nodes which are going to maximize the packet processing ability of the routing path by not exceeding the value of $Q_{t h m}$. Work selects the entries of the array $V\left[n_{n}, Q_{\text {avrg }_{n}}\right]$ by avoiding following conditions:

1) $V\left[n_{i}, Q_{\text {avrg }_{i}}\right]=\max \left(V\left[n_{i}-1, Q_{\text {avrg }_{i}}\right], E_{P_{i}}+\right.$ $\forall n_{1} \leq n_{i} \leq n_{n}$ and $0 \leq Q_{\text {avrg }_{i}} \leq Q_{\text {thm }}$

2) $V\left[0, Q_{\text {avrg }_{i}}\right]=0 \forall 0 \leq Q_{\text {avrg }_{i}} \leq Q_{\text {thm }}$

3) $V\left[n_{i}, Q_{\text {avrg }_{i}}\right]=-\infty \forall Q_{\text {avrg }_{i}} \leq 0$

To select the entries of the $V\left[n_{n}, Q_{\text {avrg }}\right]$, work uses the knapsack algorithm [22], and explained as follows;

$\operatorname{Knapsack}\left(E_{p}, n_{n}, Q_{t h m}, Q_{\text {avrg }}\right)$

\{

For $\left(Q_{\text {avrg }}=0\right.$ to $\left.Q_{\text {thm }}\right)$

$V\left[0, Q_{\text {avrg }}\right]=0$

for $\left(n_{i}=n_{i}\right.$ to $\left.n_{n}\right)$

For $\left(Q_{\text {avrg }}=0\right.$ to $\left.Q_{\text {thm }}\right)$

if $\left(Q_{\text {avrg }_{i}}\left(n_{i}\right) \leq Q_{\text {avrg }}\right)$

$V\left[n_{i}, Q_{\text {avrg }_{i}}\right]=\max \left(V\left[n_{i}-1, Q_{\text {avrg }_{i}}\right], E_{P_{i}}\right.$ $\left.+V\left[n_{i}-1, Q_{\text {avrg }_{i}}-Q_{\operatorname{avrg}_{i}}\left(n_{i}\right)\right]\right)$

Else

$V\left[n_{i}, Q_{\operatorname{avrg}_{i}}\right]=V\left[n_{i}-1, Q_{\operatorname{avrg}_{i}}\right]$

$\operatorname{returnV}\left[n_{n}, Q_{t h m}\right]$

\}

The route replay message unicast by destination through the computed path. The rest of the activities of the routing process such as sequence numbering, flag, and timer setting follows the existing routing protocols AODV [11].

\section{Performance Evaluation}

The performance of the designed routing protocol is analyzed with network simulator [12]. The simulation environment and parameters for the performance analysis are shown in Table I. We consider the 100 mobile nodes and are distributed in the wireless communication region of $1500 \mathrm{~m}^{*}$ $1500 \mathrm{~m}$. Each node consists of the 100 joules of energy and can hold the 10 packets in its buffer and the packet size consider for communication is 512 Kbytes with delay-sensitive data. The performance evaluation metrics are packet delivery, delay, and load balance efficiency and network lifetime. The performance is compared with recent existing mechanisms such as Buffer based load balancing (BDLB) [1] and energy delay-based load balancing method (EDLB)[2].

The results are shown in Fig. 4, 5, 6, and 7, and they clearly indicate that the performance of the network is enhanced by the proposed routing protocol.

Fig. 4 shows the packet delivery fraction performance in terms of increased data rates, it is clearly indicating that the proposed routing protocol deliver all the packets to the destination, as it is free from the packet drop due to either lack of energy or buffer overflow.

TABLE I. PERFORMANCE EVALUATION PARAMETERS

\begin{tabular}{|l|l|}
\hline Parameters used & values \\
\hline Time & $1200 \mathrm{~s}$ \\
\hline Nodes & 100 \\
\hline Layer & Logical Link \\
\hline MAC protocol & 802.11 \\
\hline Communication & Two-Ray Ground \\
\hline Network layer & BBLB, EDLB Proposed \\
\hline Mobility & Random-way point \\
\hline Queuing Method & Priority \\
\hline Traffic & CBR \\
\hline Energy & $100 \mathrm{~J}$ \\
\hline Network Area & $1500 \mathrm{~m} \times 1500 \mathrm{~m}$ \\
\hline
\end{tabular}

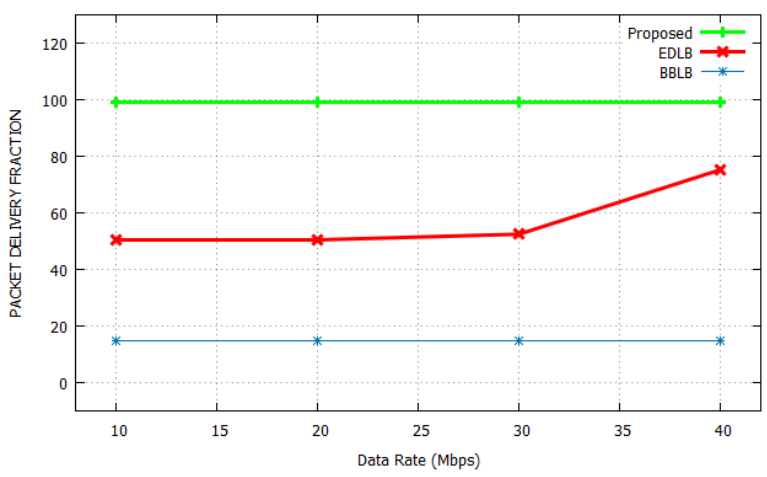

Fig. 4. Packet Delivery Fraction Performance Comparison. 
The delay of the network is low in the proposed routing protocol, as it will not consider the nodes whose transmission delay is high, and which is higher than the MAC delay. The delay comparison is shown in Fig. 5.

Fig. 6 shows the energy consumption and packet delivery fraction comparison, it is clearly mentioned that the packet delivery of the proposed routing mechanism is high with less energy consumption.

The efficiency of Load balance is calculated as the ratio of the packets balanced due to the selection of non-congested efficient node to a total number of packets. Packet delivery of a proposed routing protocol extended due to load balancing at node buffer is constructed at the proposed routing protocol, and it is shown in Fig. 7.

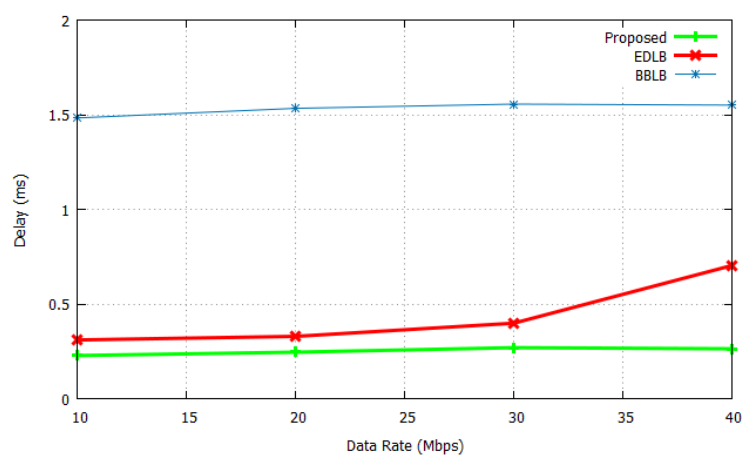

Fig. 5. End to End Delay Performance Comparison.

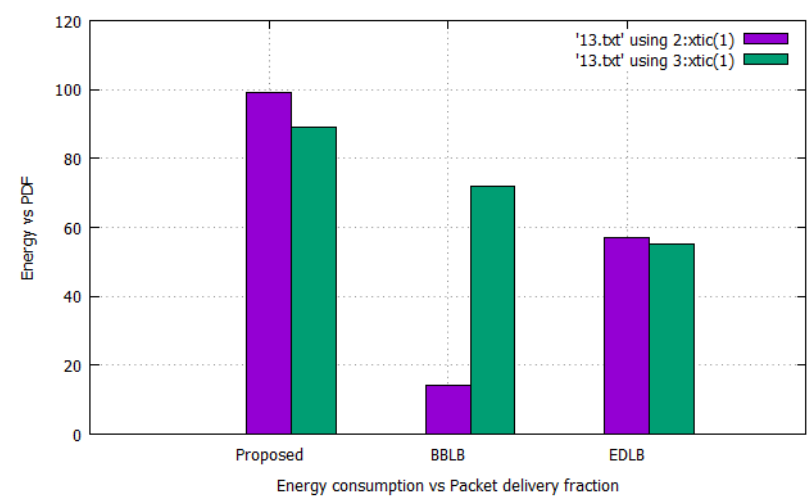

Fig. 6. Comparison between Energy Consumption vs Packet Delivery Fraction.

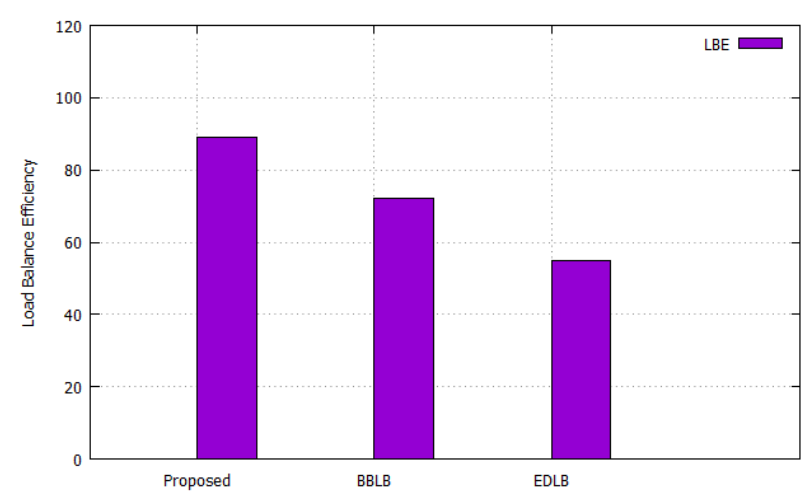

Fig. 7. Comparison of Load Balancing Efficiency.

\section{CONCLUSION}

Wireless mesh network communication gains user attractions due to its deployment flexibility. The main challenging task in the WMN is the provision of Quality of Service due to unbalanced traffic. The recent advancement in wireless technology has fueled the user's attraction towards the delay-sensitive services, which in turn additional impact on WMN to provide QoS. The paper's aim is to provide the QoS in the WMN by load balancing. In literature, various mechanisms have been designed to adder the issue, but they fail to achieve the optimal solution in terms of throughput and energy efficiency. Thus, the work designed the energy-efficient load balancing routing metric to address the limitations of existing conventional methods. Load balancing is accomplished by the selection of non-congested nodes, and energy efficiency is attained by the selection of the greatest packet processing nodes for communication. Performance results show that the proposed routing protocols outperformed in comparison with existing Buffer based load balancing, and energy delay-based load balancing routing protocols. The future work will focus to find the packets drop in the network due to malicious reasons, and further to design the routing protocol to avoid the packets drops due to malicious reasons as well as packets drop due to constrained resources issue.

\section{REFERENCES}

[1] Keerthi, D. S., and Shobha Rani Aand TG Basavaraju. "Buffer based Routing Mechanism for Load Balancing in Wireless Mesh Networks." Journal of Computer Engineering and Technology 10, no. 1 (2019): 1 10 .

[2] Keerthi, D. S., A. Shobha Rani, and T. G. Basavaraju. "Energy-Delay Based Route Request Scheme for Load Balanced Routing in Wireless Mesh Networks." In 2019 International Conference on Communication and Electronics Systems (ICCES), pp. 2052-2056. IEEE, 2019.

[3] Mohammad, Arshad Ahmad Khan, Ali Mirza Mahmood, and Srikanth Vemuru. "Intentional and unintentional misbehaving node detection and prevention in mobile ad hoc network." International Journal of Hybrid Intelligence 1, no. 2-3 (2019): 239-267.

[4] Fairhurst, Gorry, Brian Trammell, and Mirja Kühlewind. "Services provided by IETF transport protocols and congestion control mechanisms." RFC Series 8095 (2017).

[5] Hava, Adriana, Yacine Ghamri-Doudane, Gabriel-Miro Muntean, and John Murphy. "Increasing user perceived quality by selective load balancing of video traffic in wireless networks." IEEE Transactions on Broadcasting 61, no. 2 (2015): 238-250.

[6] Anbu Ananth,C., Selvakumar,K.: An efficient load balancing techniques for wireless mesh networks. Int. J. Eng. Res. Technol. 3(2), 2146-2150 (2014).

[7] Raja, G. P., and S. Mangai. "Firefly load balancing based energy optimized routing for multimedia data delivery in wireless mesh network." Cluster Computing 22, no. 5 (2019): 12077-12090.

[8] Hava, Adriana, Yacine Ghamri-Doudane, Gabriel-Miro Muntean, and John Murphy. "Increasing user perceived quality by selective load balancing of video traffic in wireless networks." IEEE Transactions on Broadcasting 61, no. 2 (2015): 238-250.

[9] Hava, Adriana, Gabriel-Miro Muntean, and John Murphy. "An energyefficient mechanism for increasing video quality of service in Wireless Mesh Networks." In 2016 IEEE Wireless Communications and Networking Conference, pp. 1-6. IEEE, 2016.

[10] Li, Hongkun, Yu Cheng, Chi Zhou, and Weihua Zhuang. "Minimizing end-to-end delay: A novel routing metric for multi-radio wireless mesh networks." In IEEE INFOCOM 2009, pp. 46-54. IEEE, 2009.

[11] Anamalamudi, Satish, Abdur Rashid Sangi, Mohammed Alkatheiri, and Ahmedin Mohammed Ahmed. "AODV routing protocol for Cognitive 
radio access based Internet of Things (IoT)." Future Generation Computer Systems 83 (2018): 228-238.

[12] Rathore, Pramod Singh, Abhishek Kumar Pandey, and Dac Nhoung Le. "Computer Network Simulation in NS2: Basic Concepts \& Protocols Implementation." (2018).

[13] Sharma, Saurabh, Dipti Jindal, and Rashi Agarwal. "Analysing Mobile Random Early Detection for Congestion Control in Mobile Ad-hoc Network." International Journal of Electrical and Computer Engineering 8, no. 3 (2018): 1305.

[14] Asami, Atena, Majid Asadi Shahmirzadi, and Sam Jabbehdari. "Multicast Routing with Load Balancing in Multi-Channel Multi-Radio Wireless Mesh Networks." International Journal of Advanced Computer Science and Applications 8, no. 12 (2017): 264-269.

[15] Dr.Mohammed Ali Hussain and S.J. Sultanuddin Token System based Efficient Route Optimization in MANET for VANET in Smart City,Transactions on Emerging Telecommunications Technologies, January 2020, Wiley Publications, Science Citation Indexed (SCI).

[16] B.Suresh babu, Dr.Mohammed Ali Hussain, Dr. Mahmood Ali Mirza Reliable And Energy Efficient Scheduling Model For Tsch Enabled Mobile Adhoc Network International Journal of Scientific \&amp; Technology Research Vol.9, Issue.1 January 2020,
[17] Dr. Mohammed Ali Hussain and Dr. Balaganesh Duraisamy Minimizing the Packets Drop by System Fault in Wireless Infrastructure less Network Due to Buffer Overflow and Constrained Energy, International Journal of Advanced Science and Technology Vol. 29, No. 5, 2020.

[18] Das, Banani, and Sudipta Roy. "Load balancing techniques for wireless mesh networks: a survey." 2013 International Symposium on Computational and Business Intelligence. IEEE, 2013.

[19] Gautam, S. and Kumar, R., 2012. A Review of Energy-Aware Routing Protocols in MANETs. International Journal of Modern Engineering Research (IJMER), 2(3).

[20] Mohammad, Arshad Ahmad Khan, Ali Mirza, and Srikanth Vemuru. "Energy aware routing for manets based on current processing state of nodes." Journal of Theoretical and Applied Information Technology 91.2 (2016): 340 .

[21] Mohammad, Arshad Ahmad Khan, Ali Mirza Mahmood, and Srikanth Vemuru. "Energy-Aware Reliable Routing by Considering Current Residual Condition of Nodes in MANETs." Soft Computing in Data Analytics. Springer, Singapore, 2019. 441-452.

[22] Martello, Silvano, and Paolo Toth. "A new algorithm for the 0-1 knapsack problem." Management Science 34.5 (1988): 633-644. 